\title{
LEAN CONSTRUCTION QUALITY ASSURANCE OPPORTUNITIES IN HIGHWAY CONSTRUCTION
}

\author{
Mohammad Moin Uddin ${ }^{1}$
}

\begin{abstract}
Quality management is a significant component of any construction project and the cost of quality can constitute a sizable part of total construction cost. US state highway agencies are in constant pressure to do more with less. Applying Lean construction management principles can be a viable way to eliminate waste and inefficiencies and maximize value and efficiency in quality assurance (QA). State highway agencies have implemented various QA practices and processes which include different QA standards and specifications, varying testing methods, central testing lab vs. on site testing, performance based vs. prescribed quality assurance practices, implementation of innovative quality assurance practices, etc. Therefore, there is an opportunity to assess different QA strategies and recommend lean QA practices that are cost effective. A survey was conducted among state highway agencies, which provided a detailed mapping of various QA practices and processes used as part of QA programs and identified areas where agencies can focus on for cost savings. The survey found that QA sampling and testing plans, optimization of sampling plans, optimization of QA standards and specifications, and implementation of innovative test methods and processes are the main areas the agencies should focus on to lean the current QA programs.
\end{abstract}

\section{KEYWORDS}

Lean construction, quality assurance, process, cost savings

\section{INTRODUCTION}

Transportation infrastructure and other transportation assets constitute some of the most important economic resources of the United States. Transportation infrastructure, also known as transportation structures, includes highways and streets, bridges, railroads, and other transportation structures. In 2016, the net value of US transportation infrastructure was estimated at $\$ 7.7$ trillion (Bureau of Transportation Statistics 2018). Having built that system and continuing to keep it efficient, Federal Highway Administration and state highway agencies' (SHA) biggest challenge in the $21^{\text {st }}$ century is to preserve the quality of our nation's top investment. The International Standard Organisation defined quality as the totality of features and characteristics of a product or a service that bear on its ability to satisfy stated or implied needs (ISO 9000 2015). Quality Assurance (QA) which are systematic actions necessary to provide confidence that a product performs

1 Associate Professor, Department of Engineering, Engineering Technology and Surveying, East Tennessee State University, Johnson City, TN 37614, USA, uddinm@etsu.edu, orcid.org/0000-0003$\underline{4259-0259}$ 
satisfactorily in service is an integral part of highway construction. When an effective construction QA program is applied, it provides confidence and assurance that the quality of workmanship and materials incorporated into the project comply with requirements and specifications of the plan.

Lean Construction extends from the objectives of a lean production systemmaximize value and minimize waste - to specific techniques, and applies them in a new project delivery process. Therefore, Lean theory, principles and techniques provide the foundation for a new form of project implementation. successful applications of lean construction principles result in reduction in cost, minimize construction time, improve safety, reliability and customer satisfaction (Babalola, Ibem, and Ezema 2019; Erol, Dikmen, and Birgonul 2016; Hosseini, Nikakhtar, Wong, and Zavichi 2011). Even though value of lean principles in construction industry is extensively studied, one area frequently overlooked is the QA program in a construction project and how applying lean principles can reduce waste and inefficiencies in it. This paper investigated various activities of a QA program and identified ways how SHA can be benefitted by applying the same lean principles.

State and federal departments of transportation realize the importance of QA and implement methods and procedures for ensuring the best quality. However, these practices differ significantly. Examples of varying QA practices are different standards and specifications, varying test methods to determine properties of a finished construction which vary in accuracy and costs, destructive vs. non-destructive tests, central testing labs vs. on-site testing, performance based vs. prescribed quality assurance practices, implementation of innovative quality assurance practices and more. Federal Highway Administration's Materials Quality Assurance provide detail descriptions of each of these practices including their strengths and limitations (FHWA 2017; Burati, Weed, Hughes, and Hill 2003, 2004; Hughes 2005). However, cost of these practices is often overlooked. The variety of approaches taken by individual states offers an opportunity to assess the cost-effectiveness of different strategies and recommend those practices that are most successful.

There is a mismatch between revenue and spending in highway trust fund, and every year, most SHAs are facing funding shortfalls or asked to do more with little. However, cost of quality activities such as cost of equipment, testing, inspection, training, etc. are significant. Numerous studies examining the cost of quality (conformance and nonconformance) have identified their costs as ranging from $5 \%$ to $20 \%$ of a project's contract value (P. Barber, Graves, Hall, and Tomkins 2000; Josephson, Larrson, and Li 2002; El-Rayes and Kandil 2005). Therefore, there is real value in identifying, sharing, and implementing cost effective quality assurance practices and procedures among SHAs. Cost of Quality consist of cost of prevention, cost of appraisal and cost of failure. Drilling down to these costs can vary widely as SHAs cost estimates vary based on location, availability of material, labor, technology, etc. (Holm, Schaufelberger, Griffin, and Cole 2005; Akinci and Fischer, 1998) It will be rather beneficial to identify various areas in existing QA practices where SHAs can further investigate using principles of lean construction which are aimed at maximizing value and minimizing costs. Therefore, the purpose of this study is to identify and share cost effective QA practices within SHAs' QA programs related to acceptance sampling and testing, optimization of QA standards and specifications, QA sampling plan optimization practices, and usage of quality measures. 


\section{COMPONENTS OF QA PROCEDURES}

Once a construction project is awarded, the contractor in consultation with the SHA prepare a QA plan for the project based on construction standards and specifications. A QA plan is formally defined as "A project-specific document prepared by the contractor which identifies all QA personnel and procedures that will be used to maintain all production and placement processes "in control" and meet the agency specification requirements" (FHWA 2012). The QA plan typically includes 1) Identification of quality characteristics (such as asphalt content, compressive strength, gradation, moisture content, etc.), 2) Sampling plan for quality control (QC) and acceptance, 3) Certification requirements of labs and lab technicians, 4) Inspections and tests including independent assurance (IA), 5) Data analysis and pay decision and 6) control of nonconformance (FHWA 2013). Most agencies require contractor QC for the majority of materials used in the construction. Many agencies retain the entire acceptance function; however, the number of agencies using contractor test results in the acceptance decision is increasing.

\section{RESEARCH METHOD AND SURVEY DESIGN}

This study utilizes an online survey and interviews with STAs' quality assurance managers focusing on current quality assurance practices used by STAs. The author developed a database of the contact information of quality assurance managers of all 50 states' transportation agencies. Online survey software "Surveymonkey" was used to develop and distribute the survey. An IRB approval was secured since human subjects were involved.

The survey was design to capture information in various aspects of a QA program. Part one of the survey questions focused on QA sampling and testing for Hot Mix Asphalt and Portland Cement Concrete Pavement. The intend of the questions were to understand typical acceptance QA tests used by STAs and their failure percentages. Part two questions requested information about SHAs' use of innovative testing methods that can potentially reduce cost, save time or produce more accurate results. Questions in part three were focused on QA practices such as sampling frequencies, standards and specifications, onsite vs. central labs, etc.

\section{DATA ANALYSIS AND RESULTS}

The survey was sent out to all 50 states. A total of 19 state highway agencies completed the survey with a response rate of 38\%. The participants range from Quality Assurance Managers to State Materials Engineers. The results of the survey are elaborated below.

\section{State Highway Agencies' Focus of Quality Performance}

Quality of construction mainly depends on the performance of material installed and the workmanship. Material performance can be measured by testing and experimenting the build characteristics of the product while workmanship is measured by using standards and codes as established benchmarks; requiring a certain level of qualifications for the trades, manufacturers, and installers involved in the project work; and acceptable tolerances of the finished work. SHAs were asked how they measure construction quality performance. About $90 \%$ of the SHAs measure both performance of workmanship and materials. Remaining $10 \%$ of the agencies measure the performance of materials only. 


\section{State Highway Agencies' Material Acceptance Testing}

To ensure quality, SHAs conduct different types of tests for materials and finished products. These tests again vary based on construction type and also from state to state. Common material testing for hot mix asphalt and Portland cement concrete pavement are describe below.

\section{Hot Mix Asphalt (HMA)}

The survey requested the state highway agencies to report about typical acceptance tests performed to measure the condition of placed HMA relative to acceptable standards/specifications. The survey found that SHAs use a wide variety of tests for acceptance. As shown in Figure 1, the in-place density test is conducted by all SHAs. About $89 \%$ of SHAs test the finished surface of HMA for smoothness. Asphalt content and air void testing are also frequently used QA tests for HMA. Other QA tests for HMA are void in mineral aggregate and void in fine aggregate, sieve analysis, and binder content.

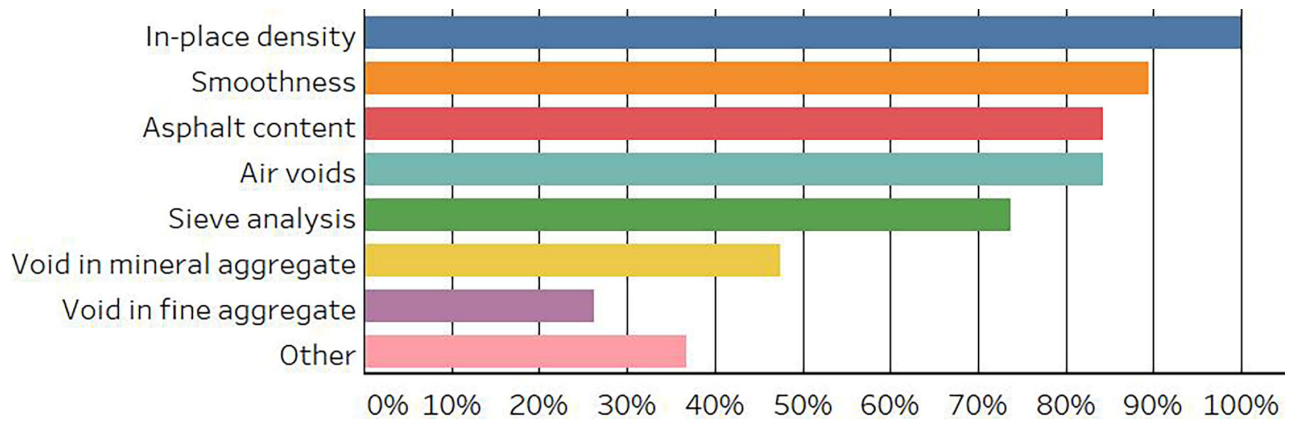

Figure 1: Typical Acceptance Tests Conducted by SHA for Hot Mix Asphalt

\section{Portland Cement Concrete Pavement (PCCP)}

SHAs were also asked about typical acceptance tests regarding PCCP. All SHAs use the compressive strength by cylinder test for acceptance, $88 \%$ utilize the air content test, and $82 \%$ utilize slump/spread and Smoothness tests (Figure 2). About $71 \%$ utilize thickness and temperature tests, and only $12 \%$ utilize the flexural strength (by cast beam) test.

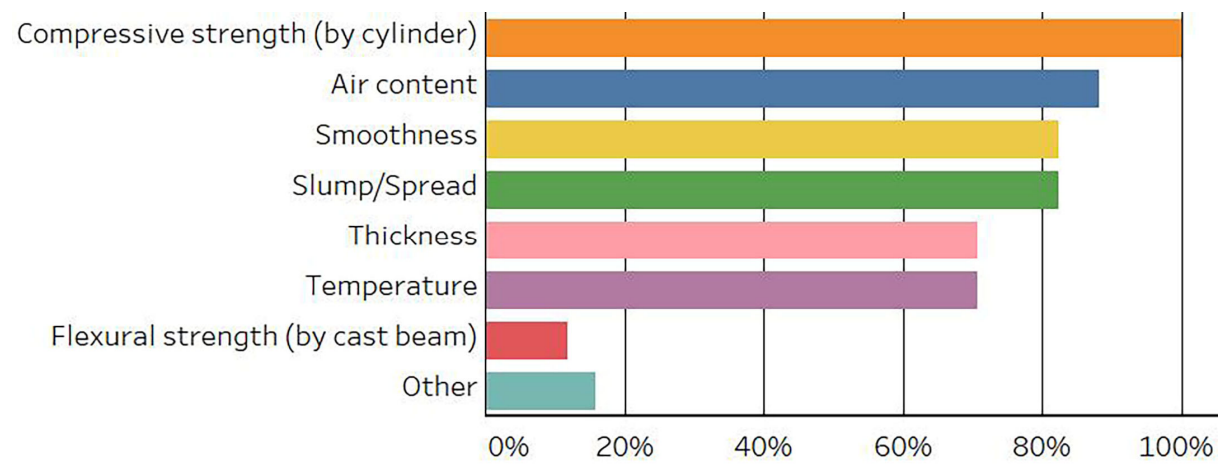

Figure 2: Typical Acceptance Tests Conducted by SHA for Portland Cement Concrete Pavement 


\section{Failure Statistics in ACCEPTAnCe Test Results}

A failure in acceptance test for highway construction is determined when the test results do not meet the specification limit for the test. For example, if a job mix formula requires an asphalt content of $4 \% \pm 1 \%$ (Lower Limit $=3 \%$ and Upper Limit $=5 \%$ ), but tests show less than $3 \%$ or more than $5 \%$ asphalt content, then it will constitute a failure according to the acceptance test, and re-working of the material related to the test is warranted until a pass is achieved. The following section summarizes failure statistics for acceptance tests for the previously mentioned five different types of materials and construction.

\section{Hot Mix Asphalt (HMA)}

The SHAs were asked to record the estimated percentage of acceptance tests failing to meet the specifications for HMA. As shown in Figure 3, for the asphalt content, 80\% responded that failures occurred only $1 \%-2 \%$ of the time; $20 \%$ responded the failures occurred $2 \%-10 \%$ of the time. Most states responded that in place density and air voids tests most frequently fail to meet the specifications ( $>2 \%$ failure frequencies), while remaining tests have less frequent failure rates ( 0 to $2 \%$ ).

\section{Portland Cement Concrete Pavement (PCCP)}

Failure to meet specification limits also occurred in PCCP acceptance testing. About $64 \%$ of SHAs reported that the air content test failed more frequently ( $>2 \%$ failure frequencies). Acceptance tests that meet specification limits more frequently are compressive strength, flexural strength, slump, and temperature (Figure 3).

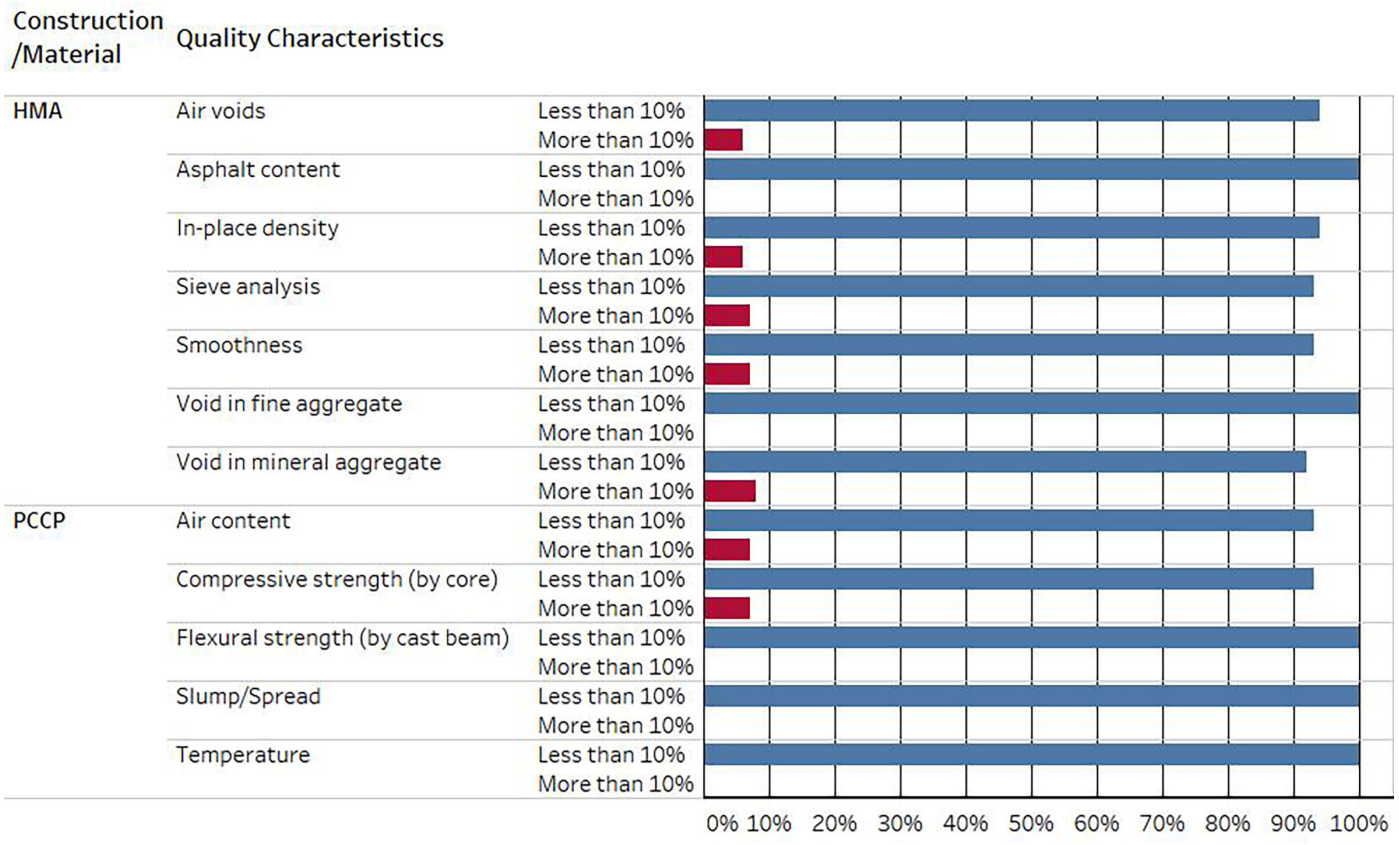

Figure 3: SHA Failures Observed in Test Results for Different Construction Types/ Materials

Based on all the tests that are conducted as part of QA acceptance tests, on average, only $5 \%$ of SHAs reported that acceptance tests fail to meet specification limits more than $10 \%$ time. This implies that out of 10 tests performed, 9 tests meet specification limits. This means that SHAs' QA standards, specifications, and sampling plans are effective and 
rigorous. The survey found that many tests have a significantly higher success rate $(>98 \%)$. This offers SHAs an opportunity to optimize specifications and sampling plans for such tests with either limiting sampling frequencies or increasing lot size for acceptance testing.

\section{Optimization of Acceptance Sampling and Testing Plan}

Most SHAs have developed QA sampling and testing plans for systematic and consistent testing of materials and finished products. SHAs consider many factors when deciding QA sampling and testing plans, however, three factors are typically considered by many SHAs. They are cost (cost of sampling and testing), risk (agency's and contractor's risk related to acceptance plan, also referred as $\alpha$ and $\beta$ risk), and importance (effects on overall construction project). There are benefits of optimizing sampling and testing plans for cost, risk, and importance. For high importance jobs where safety is a bigger factor, such as bridges, importance is the dominant factor for optimization. However, in lower importance jobs, the cost can have more weight than the risk.

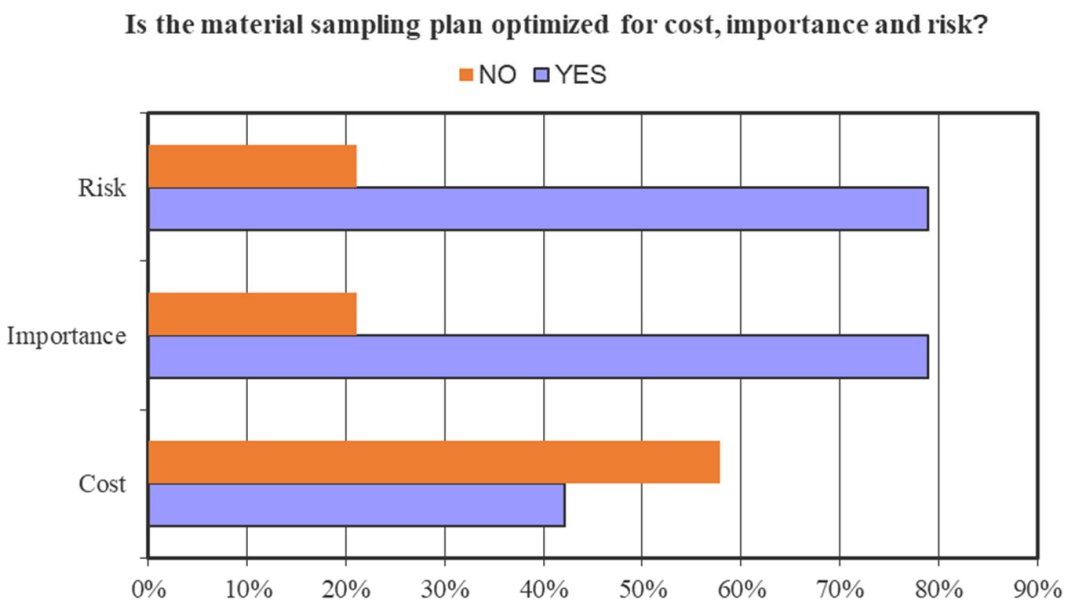

Figure 4: Optimization of Material Sampling and Testing Plan for Cost, Importance, and Risk

As shown in Figure 4, 42\% of the agencies responded that their sampling and testing plan is optimized for all of the factors: cost, risk, and importance. About $78 \%$ of the responding agencies considered either risk or importance for optimizing their sampling and testing plans. Surprisingly, cost was not as significant of a factor as importance and risk. It can be recommended that SHAs who have not optimized their sampling and testing plans for cost, risk, and importance should consider optimizing their sampling and testing plans. This would be an opportunity to reduce costs and improve efficiency in QA practices.

\section{Quality Assurance Measures for Acceptance SAMPling and TESTING}

There are several quality assurance measures that are typically used by SHAs for material and construction acceptance. These include: percent within limit (PWL), average absolute deviation (AAD), mean, statistical F\&T test, and split sample comparison. Even though FHWA recommends PWL as the quality measures to be used, other measures are still in use according to the survey. For HMA, 57\% of SHAs use PWL, 36\% use statistical F\&T test to estimate that the SHAs' tests and the contractor's tests are from the same population 
and the variability and mean of the two data sets are equal, and $71 \%$ use split sampling, which provides a check on testing equipment and procedures (Figure 5). For concrete pavement, 30\% of SHAs utilize PWL, 20\% use statistical F\&T test, and 30\% use split sampling. It needs to be mentioned that most SHAs use a combination of the above mentioned quality assurance measures for sampling and testing. However, few SHAs mentioned that they don't use any statistical method for verification test results with the contractor's test results. It is necessary to measure both the center and spread when characterizing lot material. PWL quality measure uses the mean and standard deviation to measure center and spread and then estimate the percentage of the lot that is within the specification limits. Other quality measures have limitations. For example, AAD often encourages the contractor to manipulate its process during the production of a lot in order to get the average of the test results to be at or near the target value. Split samples estimate only testing variability and can be biased if not independently obtained by SHAs or a third party. Therefore, such measures may result in acceptance of poor quality construction which may deem costly in future maintenance and rehabilitation. Implementation of PWL is the recommended cost-effective practice.

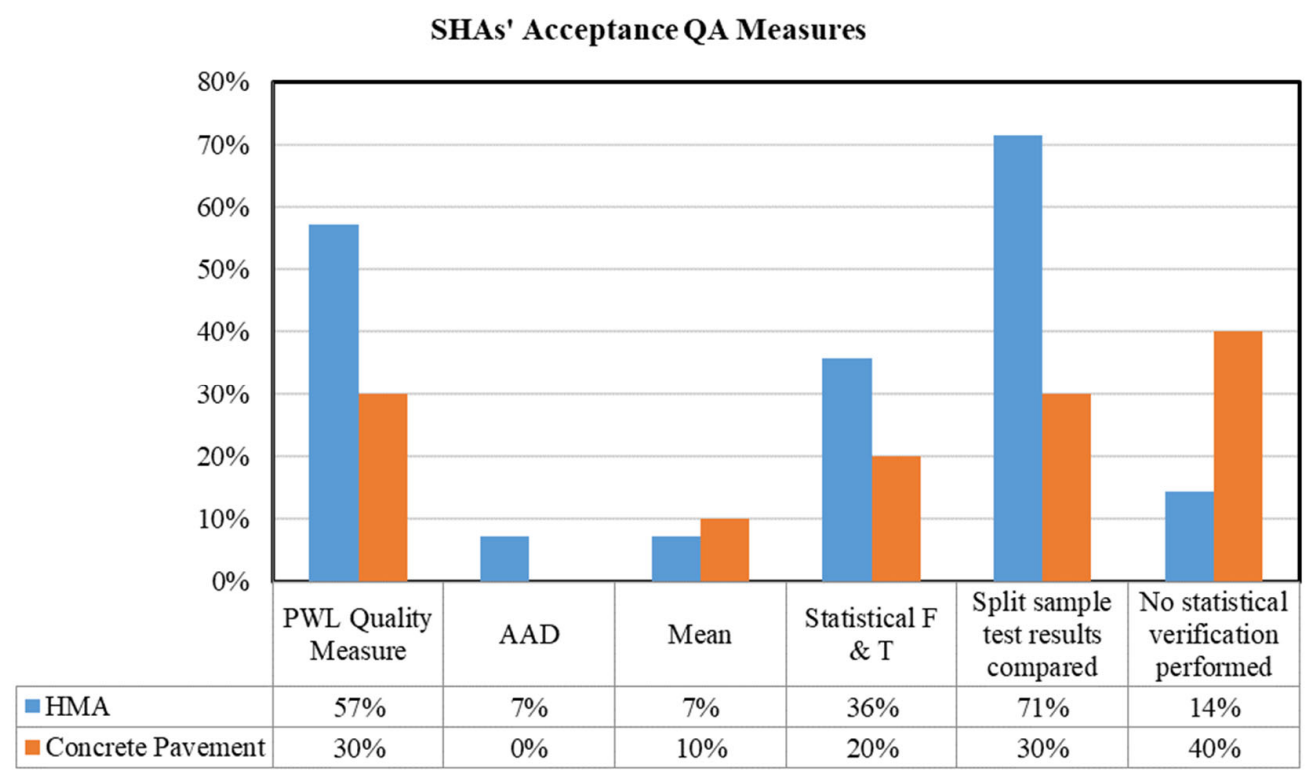

Figure 5: Frequency of SHAs Verification of Contractor Performed Quality Assurance Testing for Material Acceptance Sampling and Testing.

\section{Quality Assurance Verification Practices}

There are several methods that are used by SHAs for QA verification. They are contractor quality control sampling and testing (verified by qualified testing personnel employed by the SHAs or its designated agent, excluding the contractor and vendor), independent assurance (IA) procedures (activities that are an unbiased and independent evaluation of all the sampling and testing procedures used in the acceptance program - all agencies are required to perform IA), testing certification practices, and regional, district or divisional lab practices, etc. The survey found that, for HMA, 43\% of SHAs responded the contractor and vendor), independent assurance (IA) procedures (activities that are an unbiased and independent evaluation of all the sampling and testing procedures used in the acceptance program - all agencies are required to perform IA), testing certification 
practices, and regional, district or divisional lab practices, etc. As shown in Figure X, the survey found that, for HMA, 43\% of SHAs responded that they utilize regional, district, or divisional labs for verification testing; $14 \%$ utilize testing certification practices, $14 \%$ utilize IA procedures, and $29 \%$ utilize contractor quality control sampling and testing. For PCCP, 33\% responded that they utilize regional, district, or divisional labs for verification testing, $17 \%$ utilize testing certification practices, $17 \%$ utilize IA procedures, and $33 \%$ utilize contractor quality control sampling and testing.

\section{Use Of Innovative QUality Assurance Test Methods}

With advancements in technology come an opportunity for developing and implementing innovative quality assurance practices. In particular, quality assurance related tools and equipment are now more efficient and accurate as a result of technology improvement. As a result, many SHAs are currently using or exploring innovative testing methods (that are quick and nondestructive) that significantly reduce the cost, save time or produce more accurate results. About $58 \%$ of SHAs responded that they use innovative quality assurance test methods to reduce cost, save time, or produce more accurate results. Some of the innovate methods that SHAs are currently using or exploring for HMA are ignition oven for asphalt content, intelligent compaction, nuclear gauge and electric density gauge for in-place density, and line laser for smoothness. For PCCP, three agencies responded utilizing the maturity test for compressive strength for possible cost and time savings. For the air content test, the use of super air meters yield faster results and measures both the air void spacing and air content of fresh concrete in about 10 minutes. Air void spacing is a better indicator of concrete freeze-thaw durability than total air content. One agency is utilizing plastic air content and hardened air content to yield more accurate results.

\section{Review of Quality Assurance Practices and Procedures}

Quality assurance practices are evolving. Technological innovation, new tools and techniques, better knowledge about construction materials and finished materials, better means and methods of construction, more insight between quality assurance and construction performance, etc. are significantly impacting quality assurance practices and procedures. Only by continuously reviewing quality assurance practices, procedures, and manuals, SHAs can keep up with the latest trend which can open up new opportunities for cost and time savings. On average, 34\% of SHAs responded that they annually review quality assurance practices and procedures for: sampling frequency, acceptance limits, workmanship standards, and prescriptive work practices. About $44 \%$ of SHAs review acceptance limits annually, while only $18 \%$ of SHAs review workmanship standards annually. Most SHAs (65\%) responded that their review frequencies vary and are typically conducted on an as needed basis. Failure to review QA practices routinely can be deemed as a wasted opportunity as new technologies can be introduced which may conduct rapid data collection making old practice wasteful. It can be recommended to SHAs to review the QA practices and procedures at least annually. This would allow the state highway agencies to stay up to date with any new and innovative tools, testing methods and technology which may improve the effectiveness of QA practices and procedures.

\section{FRAMEWORK TO IMPLEMENT LEAN PRINCIPLES IN QA}

QA is an umbrella concept in highway construction which includes 1) quality planning, 2) quality control, 3) quality assurance and 4) quality improvement. Each of these steps 
involve a set of tasks and related costs. Applying lean construction principle "maximizing value and minimizing costs during project planning, design, construction and maintenance" SHAs can identify common inefficiencies and waste in QA program. SHA can apply tools and techniques namely interviews, cause and effect, diagrams, data analysis to identify inefficiency in QA program such as incorrect process and testing, waiting, unnecessary transportation, defects, etc. By systematically addressing each of these inefficiencies and waste, SHA will be able to make QA program more cost effective. A graphical presentation of the framework is illustrated in Figure 6.
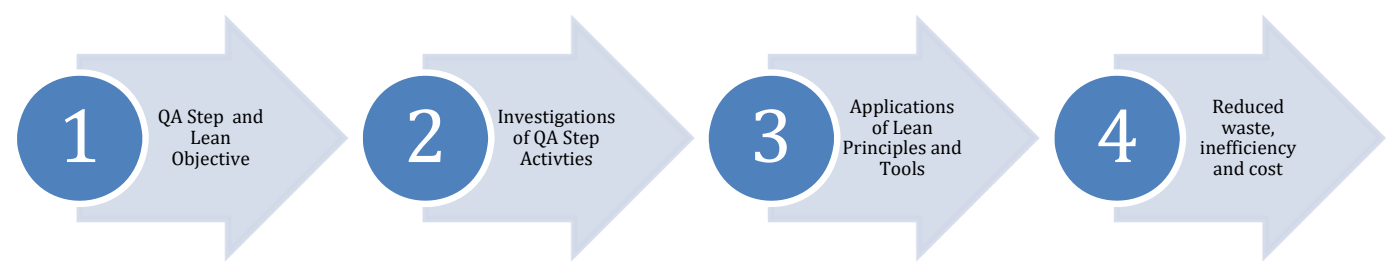

Figure 6: Framework to Implement Lean Principles in QA

\section{CONCLUSIONS}

The US highway system serves as the backbone of the country's economy. However, SHAs are constantly under pressure to do more work with less money. Because of the budget constraints, it is important to identify opportunities available within the agencies that could save costs. Successful implementation of lean construction principles in QA program can cut costs, reduce construction and increase productivity (Marosszeky, Thomas, Karim, Davis, and McGeorge 2002). The cost of quality (both conforming and nonconforming) and quality related activities can be significant. There is a real value in identifying, sharing, and implementing cost effective QA best practices and procedures among SHAs. A national electronic survey collected data that provided a detail mapping of various QA practices and processes used as part of QA program and identified areas where agencies can focus for cost savings. The main findings of the survey are 1) measurement of the performance of both material and workmanship is the best practice for ensuring quality of the construction, 2) The survey found that for many acceptance tests, more than $98 \%$ of tests meet specification limits. For such tests, there is an opportunity to optimize sampling and testing plans and related specifications and standards, 3) Even though many SHAs reported that their QA sampling and testing plans are optimized for cost, risk or importance, only few SHAs considered all three factors. About $58 \%$ of SHAs reported that cost was not considered as a factor for optimization of sampling and testing plans. A sampling and testing plan that is optimized for cost, risk, and importance will not only be efficient but cost-effective as well, 4) Percent Within Limit (PWL) is the recommended quality measure, which maximizes the value of the finished product, and 5) Use of innovative test methods, such as intelligent compaction, electronic density gauge, line laser for HMA and maturity test and super air meter for concrete are rapid and cost effective.

There are several limitations to this study. Only 19 agencies responded the survey, and the results are based on this small sample size. The survey information provided by the SHAs are opinion-based and may not be data-driven. Therefore, future studies will 
focus on the SHAs' actual acceptance data analysis and implementation of cost effective measures and quantities analysis of their cost effectiveness.

\section{REFERENCES}

Akinci, B., and Fischer, M. 1998. "Factors affecting contractors' risk of cost overburden." Journal of Management in Engineering, 14(1), 67-76.

Babalola, O., Ibem, E. O., and Ezema, I. C. 2019. "Implementation of lean practices in the construction industry: A systematic review." Building and Environment, 148, 3443

Burati, J., Weed, R. M., Hughes, C., and Hill, H. S. 2003. Optimal Procedures for Quality Assurance Specifications. Washington, DC.

Burati, J., Weed, R., Hughes, C., and Hill, H. 2004. Evaluation of Procedures for Quality Assurance Specifications, FHWA-HRT-04-046. Washington, DC.

Bureau of Transportation Statistics 2018. Value of and Investment in Transportation Infrastructure and Other Assets. Washington, DC.

Barber, P.A., Graves, M., Hall, D.S., and Tomkins, C. 2000. "Quality failure costs in civil engineering projects.” Int. J. Qual. Reliab. Manage., 17(4/5), 295-303.

El-Rayes, K., and Kandil, A. 2005. "Time-Cost-Quality Trade-off Analysis for Highway Construction." Journal of Construction Engineering and Management, 131(4), 477486.

Erol, H., Dikmen, I., and Birgonul, M.T. 2016. "Measuring the impact of lean construction practices on project duration and variability: A simulation-based study on residential buildings." Journal of Civil Engineering and Management, 23(2), 241251.

FHWA 23 CFR 637B 1995. Quality Assurance Procedures for Construction. Federal Register, Vol. 60, No. 125, p. 33712. Washington, DC.

FHWA 2012. Field Reference Manual for Quality Concrete pavements, FHWA-HIF-13059. Washington, DC.

FHWA 2013. Quality Assurance Stewardship Review Summary Report for Fiscal Years 2009 through 2012. Washington, DC.

FHWA 2017. Materials Quality Assurance. https://www.fhwa.dot.gov/pavement/qa/index.cfm (Jan 7, 2020)

Holm, L., Schaufelberger, J.E., Griffin, D., and Cole, T. 2005. Construction Cost Estimating: Process and Practices. Upper Saddle River, NJ, USA: Pearson Education.

Hosseini, S. A., Nikakhtar, A., Wong, K.Y., and Zavichi, A. 2011. "Implementing lean construction theory to construction processes' waste management." International Conference on Sustainable Design and Construction, pp. 414-420). March 23-25, Kansas City, Missouri, USA.

Hughes, C. 2005. State Construction Quality Assurance Programs. NCHRP Synthesis 346, Washington, DC.

ISO 9000. 2015. ISO 9000:2015(en) Quality management systems - Fundamentals and vocabulary. Geneva, Switzerland: The International Standard Organization.

Josephson, P., Larrson, B., and Li, H. 2002. "Illustrative benchmarking rework and rework costs in the Swedish construction industry." J. Manage. Eng, 18(2), 76-83.

Kopac, P. 2002. Making Roads Better and Better in Public Roads. Washington, DC.

Marosszeky, M., Thomas, R., Karim, K., Davis, S., and McGeorge, D. 2002. "Quality Management Tools for Lean Production - Moving From Enforcement to 
Empowerment." Proc. 10th Annual Conference of the International Group for Lean Construction, Gramado, Brazil. 
Lean Construction Quality Assurance Opportunities in Highway Construction

This page was intentionally left blank. 